\title{
Epicardial fat volume is inversely correlated with the degree of diastolic dysfunction and outcome in patients with heart failure with preserved ejection fraction
}

\author{
Gültekin Karakus*, Beatrice A Marzluf, Diana Bonderman, Jamil Babayev, Caroline Tufaro, Stefan Pfaffenberger, \\ Gerald Maurer, Julia Mascherbauer
}

From 16th Annual SCMR Scientific Sessions

San Francisco, CA, USA. 31 January - 3 February 2013

\section{Background}

Epicardial adipose tissue has been linked to cardiovascular metabolism and inflammation and has been shown to predict prevalence and progression of coronary artery disease.

The aim of the present study was to assess epicardial fat volume in patients with heart failure with preserved ejection fraction (HFPEF) in terms of quantification and predictive value.

\section{Methods}

HFPEF was defined as serum NT-proBNP levels $>220$ $\mathrm{pg} / \mathrm{ml}$, E/e by echocardiography $\geq 8$, signs or symptoms of heart failure and preserved left ventricular ejection fraction $(E F \geq 50 \%)$.

58 HFPEF patients and 34 controls were prospectively evaluated. All patients underwent right heart catheterization. CMR studies included the assessment of cardiac function and dimensions by standard cine sequences. Epicardial fat volume was quantified offline, using dedicated software $\left(\mathrm{cmr} 42^{\circledR}\right)$.

\section{Results}

Epicardial fat volume ranged from 23 to $89 \mathrm{ml}$ (mean 49.3 $\pm 16.2 \mathrm{ml}$; patients: $43.8 \pm 13.4 \mathrm{ml}$, controls: $58.6 \pm 16.6 \mathrm{ml}$; $\mathrm{p}<0.001)$. Epicardial fat volume was significantly correlated with $E / e^{\prime}(R=-0.37 ; p<0.001)$, NT-proBNP $(R=-0.27$; $\mathrm{p}=0.012)$, right ventricular size and function $(\mathrm{R}=-0.32$; $\mathrm{p}=0.002$ and $\mathrm{R}=0.40 ; \mathrm{p}<0.001)$, left ventricular ejection fraction $(R=0.36, p<0.001)$, left and right atrial size $(R=-0.27$, $\mathrm{p}=0.01 ; \mathrm{R}=-0.34 ; \mathrm{p}=0.001)$, mean pulmonary arterial pressure $(R=-0.36, p=0.006)$, pulmonary capillary wedge pressure $(\mathrm{R}=-0.33 ; \mathrm{p}=0.013)$, and pulmonary vascular resistance $(\mathrm{R}=-0.34 ; \mathrm{p}=0.01)$. Epicardial fat volume was not correlated with gender, age, renal function, or body mass index.

All study participants were followed for $356 \pm 198$ days. By Kaplan-Meier analysis, event-free survival was significantly worse in subjects with epicardial fat volume below the median of $43 \mathrm{ml}(\log$ rank $\mathrm{p}=0.038)$.

\section{Conclusions}

Epicardial fat volume is inversely correlated with diastolic dysfunction, serum NT-proBNP, invasive measures of pulmonary hypertension, but not total body fat. Decreasing epicardial fat volume predicts adverse outcome in HFPEF patients. The mechanism causing decreasing epicardial fat volume in advanced disease remains to be determined.

\section{Funding}

none.

Published: 30 January 2013

doi:10.1186/1532-429X-15-S1-P268

Cite this article as: Karakus et al.: Epicardial fat volume is inversely correlated with the degree of diastolic dysfunction and outcome in patients with heart failure with preserved ejection fraction. Journal of Cardiovascular Magnetic Resonance 2013 15(Suppl 1):P268. 